\title{
A176 MYOSTATIN - A NEW PLAYER IN INFLAMMATORY BONE LOSS
}

B Dankbar, ${ }^{1}$ C Wunrau, ${ }^{1}$ C Wehmeyer, ${ }^{1}$ T Pap $^{1}{ }^{1} /$ nstitute of Experimental Musculoskeletal Medicine - IEMM, University Hospital Muenster, Muenster, Germany

\subsection{6/ard.2010.149013.19}

Rheumatoid arthritis (RA) is the prototype for an inflammatory arthritis, characterised by chronic inflammation and progressive joint destruction. In this context, tumour necrosis factor $\alpha(T N F \alpha)$ is a key cytokine in promoting destruction of bone by increasing the number of bone-resorbing osteoclasts and decreasing the number of bone-forming osteoblasts, thereby leading to an overall bias toward bone resorption. Myostatin (GDF-8) is a member of the transforming growth factor $\beta$ family that is expressed in skeletal muscle, which is also its primary target tissue. Since several lines of evidence suggest a role for myostatin also in bone homeostasis, the authors investigated the specific contribution of myostatin to pathological bone remodelling and damage in RA.

Methods In vivo, myostatin expression was studied by immunohistochemistry in synovial tissues of RA and osteoarthritis (OA) patients as well as in arthritic human TNF transgenic (hTNFtg) mice. Effects of myostatin on osteoclast differentiation were evaluated by tartrate-resistant acid phosphatase (TRAP) staining and signalling pathways as well as expression of osteoclast-specific genes were analysed by immunoblotting. To assess the functional role of myostatin in vivo, myostatin knockout $\left(\mathrm{Mstn}^{-/-}\right)$mice were crossed with hTNFtg mice and the clinical severity of disease was assessed. In addition, histological changes including bone erosion and inflammation were evaluated by histomorphometric analyses.

Results Myostatin was highly expressed in RA synovial tissues with most prominent staining of synovial fibroblasts. In contrast, only very few myostatin expressing cells were found in OA synovial tissues. In line with these results, strong expression of myostatin in joints of hTNFtg mice was observed, whereas only negligible staining was found in wild type animals. In vitro, myostatin dramatically accelerated receptor activator of nuclear factor $\kappa B$ ligand (RANKL)-induced osteoclast development. TRAP staining revealed the appearance of very large osteoclasts with 
a huge cytoplasmatic compartment and a very high number of nuclei already after 3 days. In contrast, the conventional setting of osteoclast differentiation (macrophage colonystimulating factor and RANKL) did not generate osteoclasts during this short time period. In addition, myostatin-deficient BMMs showed a reduced RANKL-induced osteoclast formation, suggesting that myostatin is not only a paracrine stimulator of osteoclastogenesis. Histomorphometric analyses revealed that the lack of myostatin not only ameliorate the clinical severity of arthritis in hTNFtg mice but improve joint damage in this mouse model of RA. Mstn ${ }^{-/} /$hTNFtg mice displayed less bone erosion and synovial inflammation compared to hTNFtg mice.

Conclusions This data suggest that the expression of myostatin in arthritic joints is associated with an enhanced osteoclast formation and thus, significantly involved in bone destruction during chronic inflammatory arthritis. 